\title{
Isospin Symmetry Breakings as from QCD Sum Rules
}

\author{
W-Y. Pauchy Hwanga* \\ Department of Physics, National Taiwan University, Taipei, Taiwan 10764 \\ Center for Theoretical Physics, Laboratory for Nuclear Science and \\ Department of Physics, Massachusetts Institute of Technology, \\ Cambridge, Massachusetts 02139
}

We investigate isospin symmetry breakings, especially those connected with pion-nucleon couplings and the neutron-proton mass difference, by making use of the method of QCD sum rules in the presence of an external pion field. Manifestations of such isospin symmetry breakings are examined briefly in relation to low-energy nucleon-nucleon ${ }^{1} S_{0}$ scatterings. We discuss the prospect of understanding the various isospin symmetry breaking phenomena observed in low-energy nuclear physics by extending chiral perturbation theory $(\chi \mathrm{PT})$ to include isospin symmetry breakings (ISB). We emphasize that QCD sum rules help to establish $\chi \mathrm{PT} / \mathrm{ISB}$ out of QCD by eliminating an excessive number of ISB condensate parameters, making $\chi \mathrm{PT} / \mathrm{ISB}$ a worthy extension of $\chi \mathrm{PT}$.

\section{Introduction}

Isospin symmetry and its possible violations constitute an integral part of nuclear physics [1], not only in the sense that good symmetry offers an effective classification of nuclear or hadronic structure but also in the proximity of the isospin symmetry concept to other fundamental ideas such as chiral symmetry. In general, isospin symmetry breaking effects observed in nuclear physics may arise from different sources such as, in addition to the well known electromagnetic interactions, strong interactions but augmented with hadron mass differences (such as the $n-p$ or $\pi^{ \pm}-\pi^{0}$ mass difference) or with different meson-nucleon couplings (such as non-universal $\pi N N$ couplings) or with isospin mixings (such as $\rho-\omega$ or $\pi-\eta$ mixing). Determination of isospin symmetry breaking effects requires information on quantities which may not be directly accessible from experiments, such as the meson-nucleon couplings or the isospin mixing parameters. Accordingly, reliable theoretical predictions become indispensible for advancing our understanding of the field.

A quantitative treatment of isospin symmetry breakings often involves the capability of treating strong interactions at a quantitative level or, in other words, the ability to handle the complications due to quantum chromodynamics (QCD) in a reliable manner. To some extent, the method of QCD sum rules, as proposed originally by Shifman, Vain-

\footnotetext{
"The author wishes to acknowledge Kwei-Chou Yang, Shi-lin Zhu, and Ernest M. Henley for collaborations and discussions related to the subject presented here. This work was supported in part by the National Science Council of R.O.C. (NSC86-2112-M002-010Y).
} 
shtein, and Zakharov [2] and adopted, or extended, by many others [3-5], incorporates the nonperturbative QCD effects through various condensates (associated with the nontrivial QCD vacuum), thereby offering some hope of being able to treat strong interactions in a quantitative manner.

We wish in this work to focus on the determination of isospin symmetry breakings in pion-nucleon couplings, with some emphasis on manifestations of such symmetry breaking in low-energy nucleon-nucleon ${ }^{1} S_{0}$ scattering. We discuss briefly the prospect of understanding the various isospin symmetry breaking phenomena observed in low-energy nuclear physics by extending chiral perturbation theory $(\chi \mathrm{PT})$ to include isospin symmetry breakings (ISB). We emphasize that QCD sum rules help to establish $\chi \mathrm{PT} / \mathrm{ISB}$ out of QCD by eliminating an excessive number of ISB condensate parameters, making $\chi \mathrm{PT} / \mathrm{ISB}$ a worthy extension of $\chi \mathrm{PT}$.

\section{Isospin Symmetry Breakings in Pion-Nucleon Couplings}

In the method of QCD sum rules in the presence of an external field, we attempt to evaluate, both at the quark and hadron levels, the correlation function specified by

$\left.\Pi(p) \equiv i \int d^{4} x e^{i p \cdot x}\left(0\left|T\left(\eta_{p}(x) \bar{\eta}_{p}(0)\right)\right| 0\right\rangle\right|_{\pi}$,

where we have

$$
\begin{aligned}
& \eta_{p}(x)=\epsilon^{a b c}\left\{u^{a T}(x) C \gamma_{\mu} u^{b}(x)\right\} \gamma^{5} \gamma^{\mu} d^{c}(x) \\
& \bar{\eta}_{p}(y)=\epsilon^{a b c}\left\{\bar{u}^{b}(y) \gamma_{\nu} C \bar{u}^{a T}(y)\right\} \bar{d}^{c}(y) \gamma^{\nu} \gamma^{5}
\end{aligned}
$$

By evaluating the appropriate correlation function in the presence of the external pion field, we may determine the pion-nucleon couplings $g_{\pi^{0} p p}, g_{\pi^{0} n}$, and $g_{\pi^{+} p n}$. An alternative method is to evaluate correlation funtions with $T$ product of currents sandwiched between the vacuum and the one-pion state, as first suggested by [6] and recently adopted by some authors [7]. It appears that the induced condensates involved in the external field method are now well understood and it becomes relatively straightforward to perform calculations to higher dimensions (as required for reliable predictions). This is what we have chosen to obtain isospin symmetry breakings in $\pi N N$ couplings.

The above correlation function allows us to determine the $\pi^{0} p p$ coupling. At the quark level, we use

$$
\left\langle 0\left|T \eta_{p}(x) \bar{\eta}_{p}(0)\right| 0\right\rangle_{\pi^{0}}=2 \mathrm{i} \epsilon^{a b c} \epsilon^{a^{\prime} b^{\prime} c^{\prime}} \operatorname{Tr}\left\{S_{u}^{b b^{\prime}}(x) \gamma_{\nu} C\left[S_{u}^{a a^{\prime}}(x)\right]^{T} C \gamma_{\mu}\right\} \gamma_{5} \gamma_{\mu} S_{d}^{c c^{\prime}}(x) \gamma_{\nu} \gamma_{5}
$$

Here the quark propagator is given by

$S(x)=S^{(0)}(x)+S^{(\pi)}(x)$,

with

$$
\begin{aligned}
\mathrm{i} S^{(0) a b}(x)= & \frac{\mathrm{i} \delta^{a b}}{2 \pi^{2}{ }^{4}} \hat{x}+\frac{\mathrm{i}}{32 \pi^{2} x^{2}} \frac{\lambda_{a b}^{n}}{2} g_{c} G_{\mu \nu}^{n}\left(\sigma^{\mu \nu} \hat{x}+\hat{x} \sigma^{\mu \nu}\right)-\frac{\delta^{a b}}{12}\langle\bar{q} q) \\
& +\frac{\delta^{a b} x^{2}}{192}\left\langle g_{c} \bar{q} \sigma \cdot G q\right\rangle-\frac{m_{q} \delta^{a b}}{4 \pi^{2} x^{2}}+\frac{m_{q}}{32 \pi^{2}} \frac{\lambda_{a b}^{n}}{2} G_{\mu \nu}^{n} \sigma^{\mu \nu} \ln \left(-x^{2}\right) \\
& -\frac{\delta^{a b}\left\langle g_{c}^{2} G^{2}\right\rangle}{2^{9} \times 3 \pi^{2}} m_{q} x^{2} \ln \left(-x^{2}\right)+\frac{i \sigma^{a b m_{q}}}{48}\langle\bar{q} q) \hat{x}-\frac{1}{2^{7} \times 3^{2}} i m_{q}\left\langle g_{c} \bar{q} \sigma \cdot G q\right\rangle \delta^{a b} x^{2},
\end{aligned}
$$

and 


$$
\begin{aligned}
\mathrm{i} S^{(\pi) a b}(x)= & -\frac{\mathrm{i} \delta^{a b}}{4 \pi^{2}{ }^{2}} g_{q} \vec{\tau} \cdot \vec{\pi} \gamma_{5}+\frac{\mathrm{i} \delta^{a b}}{24} g_{q} \vec{\tau} \cdot \vec{\pi} \gamma_{5} \chi\langle\bar{q} q\rangle \\
& -\frac{\mathrm{i} \delta^{a b}}{384} m_{0}^{\pi} g_{q} \vec{\tau} \cdot \vec{\pi} \gamma_{5}\langle\bar{q} q) x^{2}+\frac{i}{2^{5} \pi^{2}} g_{c} g_{q} \vec{\tau} \cdot \vec{\pi} \gamma_{5} \frac{\lambda_{a b}^{n}}{2} G_{\mu \nu}^{n} \sigma^{\mu \nu} \ln \left(-x^{2}\right) \\
& -\frac{\mathrm{i}^{\circ b}}{2^{\circ} \times 3 \pi^{2}} g_{q} \vec{\tau} \cdot \vec{\pi} \gamma_{5}\left\langle g_{c}^{2} G^{2}\right\rangle x^{2} \ln \left(-x^{2}\right)-\frac{\sigma^{a b}}{48} g_{q} \vec{\tau} \cdot \vec{\pi} \gamma_{5}\langle(\vec{q} q) \hat{x},
\end{aligned}
$$

where $\hat{x} \equiv x_{\mu} \gamma^{\mu}$, and we have also introduced $\left\langle\bar{\psi}(0) i \gamma^{5} \tau^{j} \psi(0)>\equiv g_{q} \chi \pi^{j}\langle\bar{q} q\rangle\right.$, and $<\bar{\psi}(0) i \gamma^{5} \tau^{j} g_{c} \sigma \cdot G \psi(0)>\equiv g_{q} m_{0}^{\pi} \tau^{j}<\bar{q} q>$ with $\psi$ the isospin doublet consisting of $u$ and $d$ quarks.

We also note that, in the case of the $\pi^{-} p n$ or $\pi^{+} n p$ coupling, we use

$$
\left\langle 0\left|T \eta_{p}(x) \bar{\eta}_{n}(0)\right| 0\right\rangle_{\pi^{-}}=-4 \mathrm{i} \epsilon^{a b c} \epsilon^{a^{\prime} b^{\prime} c^{\prime}} \gamma_{\mu} \gamma_{5} S_{d}^{a a^{\prime}}(x) \gamma_{\nu} C\left[S^{(\pi) b b^{\prime}}(x)\right]^{T} C \gamma_{\mu} S_{u}^{c c^{\prime}}(x) \gamma_{5} \gamma_{\nu}
$$

We note the absence of $i S^{(0)}(x)$ in the present case, due to a change in the electric charge. The structure (which does not involve the trace of some product) seems quite different from the $\pi^{0} p p$ case, but it can be shown [8] that isospin symmetry is exact should we assume $m_{u}=m_{d}$ and $<\bar{u} u>=<\bar{d} d>$.

On the other hand, we may parametrize the correlation functions at the hadronic level in the standard manner.

$$
\begin{aligned}
& \left.\int d^{4} x\left\langle 0\left|T \eta_{p}(x) \bar{\eta}_{n}(0)\right| 0\right\rangle\right|_{\pi^{-}}=\lambda_{n} \lambda_{p} \frac{\mathrm{i}}{\hat{\mathrm{p}}-\mathrm{m}_{\mathrm{p}}}\left(-\gamma_{5} \sqrt{2} g_{\pi^{-} p n}\right) \frac{\mathrm{i}}{\hat{\mathrm{p}}-\mathrm{m}_{\mathrm{n}}}+\cdots, \\
& \left.\int d^{4} x\left\langle 0\left|T \eta_{p}(x) \bar{\eta}_{p}(0)\right| 0\right\rangle\right|_{\pi^{0}}=\lambda_{p}^{2} \frac{\mathrm{i}}{\hat{\mathrm{p}}-\mathrm{m}_{\mathrm{p}}}+\lambda_{p}^{2} \frac{\mathrm{i}}{\hat{\mathrm{p}}-\mathrm{m}_{\mathrm{p}}}\left(-\gamma_{5} g_{\pi^{0} p p}\right) \frac{\mathrm{i}}{\hat{\mathrm{p}}-\mathrm{m}_{\mathrm{p}}}+\cdots
\end{aligned}
$$

In this way, we obtain [8] the following QCD sum rule for the $\pi^{0} p p$ coupling:

$$
\begin{aligned}
& M^{6} L^{-4 / 9} E_{2}-\frac{\chi}{2} a_{d} M^{4} L^{2 / 9} E_{1}-\frac{11}{24} b M^{2} E_{0}+\frac{4}{3} a_{u}^{2} L^{4 / 9} \\
& +\frac{m_{0}^{2}}{3} a_{u}^{2} \frac{1}{M^{2}} L^{-2 / 27}+m_{0}^{2} \ln \frac{M^{2}}{\mu^{2}} L^{-26 / 27}\left[\frac{7}{8}\left(a_{u} m_{d}+a_{d} m_{u}\right)-\frac{1}{4} m_{u} a_{u}\right] \\
& +m_{0}^{2} L^{-26 / 27}\left[\frac{5}{12} m_{u} a_{u}-\frac{1}{8} m_{d} a_{d}+\frac{67}{48} m_{d} a_{u}+\frac{115}{48} m_{u} a_{d}\right] \\
= & \frac{g_{\pi^{0} \mathrm{pp}}}{g_{\pi^{0} d d}} \tilde{\lambda}_{p}^{2} e^{-\frac{m_{p}^{2}}{M^{2}}},
\end{aligned}
$$

with $a_{q} \equiv-(2 \pi)^{2}<\bar{q} q>,<\bar{q} \sigma \cdot G>\equiv-m_{0}^{2}<\bar{q} q>$ and $b \equiv<g_{c}^{2} G^{2}>$. $M$ is the Borel mass and $\tilde{\lambda}_{p}^{2}=(2 \pi)^{4} \lambda_{p}^{2}$. Analogously, we obtain the QCD sum rule for the $\pi^{+} n p$ coupling:

$$
\begin{aligned}
& M^{6} L^{-4 / 9} E_{2}-\frac{\chi}{2} \bar{a} M^{4} L^{2 / 9}-\frac{11}{24} b M^{2} E_{0}+\left\{\frac{4}{3} a_{u} a_{d}+\frac{1}{3}\left(a_{d}-a_{u}\right) a_{d}\right\} L^{4 / 9} \\
& -\left(m_{u}-m_{d}\right) a_{u} M^{2} L^{-4 / 9} E_{0}+m_{0}^{2} L^{-26 / 27}\left\{-\frac{1}{2} a_{u} m_{u}-\frac{5}{24} a_{d} m_{d}+\frac{29}{12} a_{u} m_{d}+\frac{19}{8} a_{d} m_{u}\right\} \\
& +m_{0}^{2} \ln \frac{M^{2}}{\mu^{2}} L^{-26 / 27}\left\{a_{u} m_{d}+a_{d} m_{u}-\frac{1}{4} m_{u}\left(a_{d}+a_{u}\right)\right\}+\frac{m_{0}^{2}}{3} a_{u} a_{d} L^{-2 / 27} \frac{1}{M^{2}} \\
= & \frac{g_{\pi}+n p}{g_{\pi}+d u}(2 \pi)^{4} \lambda_{n} \lambda_{p} e^{-\frac{m^{2}}{M^{2}}} .
\end{aligned}
$$

Here $E_{0}=1-e^{-x}, E_{1}=1-(1+x) e^{-x}$, and $E_{2}=1-\left(1+x+\frac{x^{2}}{2}\right) e^{-x}$ with $x=W / M^{2}$, and $L=0.621 \ln (10 M)$.

There are several sources for isospin symmetry breakings, viz.:

(1) At the hadronic level (r.h.s.), we have $m_{n} \neq m_{p}, \lambda_{n} \neq \lambda_{p}$, and $W_{n} \neq W_{p}$ (with $W$ the threshold parameter in the continuum approximation for treating the excited states and the continuum). Apart from the well known neutron-proton mass difference, we use the parameters previously adopted by Yang et al. [9]. 
(2) At the quark level (1.h.s.), the isospin symmetry breakings caused by $<\bar{d} d>\neq<$ $\bar{u} u>$ and $m_{d} \neq m_{u}$ have been made explicit in the formulae given above. In addition, there is an effect due to the non-universal pion-quark couplings. This gives rise to an important isospin symmetry breaking effect which has been taken into account in [8]. We finally obtain [8]

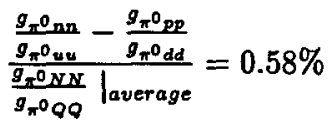

$$
\begin{aligned}
& \frac{\frac{g_{\pi+n P}}{g_{\pi+d u}}-\frac{g_{\pi^{0}} p P}{g_{\pi^{0} d d}}}{\left.\frac{g_{\pi^{0}} N N}{g_{\pi^{0}} \diamond Q}\right|_{\text {average }}}=0.35 \%
\end{aligned}
$$

Combining with the difference in pion-quark couplings (from vertex renormalizations), we conclude that $g_{\pi^{0} n n}$ is numerically bigger than $g_{\pi^{0} p p}$ by $0.80 \%$, while $g_{\pi^{+} n p}$ is numerically greater than $g_{\pi^{0} p p}$ by $0.15 \%$.

We proceed to note that the ${ }^{1} S_{0}$ phase shift $\delta_{0}$, as used to describe low-energy nucleonnucleon scatterings, can be parametrized in the standard manner:

$k \cot \delta_{0}=-\frac{1}{a}+\frac{1}{2} k^{2} r_{0}+\ddots$,

with $a$ the scattering length and $r_{0}$ the effective range. Experimentally, we have [10]

$$
\begin{aligned}
& \delta a_{\mathrm{CSB}} \equiv\left|a_{p p}\right|-\left|a_{n n}\right|=-(1.5 \pm 0.5) f m \\
& \delta a_{\mathrm{CD}} \equiv\left|a_{n p}\right|-\left|a_{n n}\right|=(5.0 \pm 0.3) f m .
\end{aligned}
$$

As already noted in [11], the differences in OPEP's, such as $V_{p p}(r)-V_{n n}(r)$, may be treated as a perturbation, making it easy to compute $\delta a_{\mathrm{CSB}}$ or other low-energy isospin symmetry breaking observables. In this way, our prediction on the difference between $g_{\pi^{0} n n}$ and $g_{\pi^{0} p p}$ gives rise to about $-1.0 \mathrm{fm}$ on $\delta a_{\mathrm{CSB}}$, consistent with the obervation both in sign and roughly in magnitude.

As already known $[1,11]$ for some time, the bulk of the charge-dependent effect as revealed by a (relatively) large value of $\delta a_{\mathrm{CD}}$ can be understood quantitatively as caused by the pion mass difference $m_{\pi^{+}} \neq m_{\pi^{0}}$ while the differences as found in pion-nucleon couplings give rise to relatively small contribution to $\delta a_{\mathrm{CD}}$. As a result, we may safely conclude that the observed $\delta a_{\mathrm{CSB}}$ and $\delta a_{\mathrm{CD}}$ as summarized recently in [10] can be understood, to a large extent, as manifestations of isospin asymmetries associated with the one-pion exchange potential.

\section{Discussion and Summary}

It may useful to mention that charge-symmetry breakings observed in medium-energy elastic $n-p$ scattering (at TRIUMF and IUCF) may be dictated primarily [12] by the charge symmetry breaking force as caused by the $\rho-\omega$ mixing or by $g_{\omega p p} \neq g_{\omega n n}$. However, the short-range nature in this case may require a careful investigation of the quarkinterchange mechanisms as addressed in [13], as it is difficult to reconcile the picture of exchanging, between two nucleons, a meson ( $\rho$ or $\omega$ ) with a range of about 0.25 fermi, 
considerably smaller than the extent of a nucleon (with a radius of at least 0.5 fermi, even assuming a little bag picture for the nucleon).

Nevertheless, the isospin symmetry breakings associated with the one-pion exchange potential (which is clearly on a very sound conceptual ground) can best be accessed through careful measurements of low-energy scattering parameters such as $\delta a_{\mathrm{CSB}}, \delta a_{\mathrm{CD}}$, etc. Such efforts should be further encouraged by the currently popular wisdom that the low-energy sector of the $\pi N$ are $N N$ systems can be described by the long-wave length limit of QCD via Goldstone theorem, yielding the so-called chiral perturbation theory $(\chi \mathrm{PT})$, so that the low-energy scattering parameters seems to be more "fundamental" than those needed in, e.g., the description of medium-energy nucleon-nucleon scatterings. In this connection, it is of importance to emphasize that QCD sum rules help to establish $\chi \mathrm{PT} / \mathrm{ISB}$ out of QCD by eliminating an excessive number of ISB condensate parameters, making $\chi \mathrm{PT} / \mathrm{ISB}$ a worthy extension of $\chi \mathrm{PT}$.

\section{REFERENCES}

1. E.M. Henley and G. A. Miller, in Mesons in Nuclei, edited by M. Rho and D.H. Wilkinson (North-Holland, Amsterdam, 1979).

2. M.A. Shifman, A.I. Vainshtein, and V.I. Zakharov, Nucl. Phys. B 147 (1979) 385, $448,519$.

3. L.I. Reinders, H.R. Rubinstein, and S. Yazaki, Nucl. Phys. B196 (1982) 125; L.I. Reinders, H.R. Rubinstein, and S. Yazaki, Phys. Rep.127 (1985) 1.

4. B.L. Ioffe, Nucl. Phys. B188, 317 (1981); [E] B191 (1981) 591; V.M. Belyaev and B.L. Ioffe, Zh. Eksp. Teor. Fiz. 83 (1982) 876 [Sov. Phys. JETP56 (1982) 493]; B.L. Ioffe and A.V.Smilga, Phys. Lett. B114 (1982) 353; B.L. Ioffe and A.V. Smilga, Nucl. Phys. B232 (1984) 109.

5. I.I.Balitsky and A.V.Yung, Phys. Lett. B129 (1983) 328.

6. N.S. Craigie and J. Stern, Nucl. Phys. B216 (1983) 209.

7. M.C. Birse and B. Krippa, U. Manchester Preprint MC/TH 96/19.

8. W-Y. P. Hwang, Ze-sen Yang, Y.S. Zhong, Z.S. Zhou, and Shi-lin Zhu, Phys. Rev. C, submitted for publication (nucl-th/9610025).

9. K.C. Yang, W-Y. P. Hwang, E.M. Henley, and L.S. Kisslinger, Phys. Rev. D47 (1993) 3001.

10. G.A. Miller et al., Phys. Rep. 194 (1990) 1.

11. Z.-J. Cao and W-Y. P. Hwang, Phys. Rev. C29 (1984) 1.

12. S. Gardner, C.J. Horowitz, and J. Piekarewicz, Phys. Rev. C53 (1996) 1143.

13. W-Y. P. Hwang, Ann. Phys. (New York) 174 (1987) 247. 